\title{
GENERACIÓN, DIFUSIÓN Y TRANSFERENCIA DEL CONOCIMIENTO DE ENFERMERÍA A LA PRÁCTICA DEL CUIDADO
}

\section{GENERATION, DIFFUSION AND TRANSFER OF NURSING CARE KNOWLEDGE INTO PRACTICE}

\author{
María Elena Lagos-Garrido* \\ Tatiana Paravic-KlijN ${ }^{* *}$
}

\begin{abstract}
RESUMEN
El objetivo de este artículo es analizar y reflexionar sobre los aspectos relacionados con la generación, difusión y transferencia del conocimiento de enfermería a la práctica del cuidado. La generación del conocimiento de enfermería ha evolucionado gracias al desarrollo de la investigación científica, la cual ha producido conocimiento para dar respuestas a las necesidades de la profesión y de la sociedad, la difusión del mismo es imprescindible para que se produzca la transferencia de este conocimiento a la práctica de enfermería; sin embargo existen limitaciones y problemas en estas etapas del proceso que deben ser superados para lograr que este conocimiento permita ser contrastado con los cuidados proporcionados a las personas, familias y comunidades de la sociedad humana.
\end{abstract}

Palabras clave: Enfermería, generación del conocimiento, difusión del conocimiento, gestión del conocimiento, traducción del conocimiento.

\begin{abstract}
The aim of this paper is to analyze and meditate about the aspects related to the generation, dissemination and knowledge transfer to nursing care practice. The nursing knowledge generation has evolved thanks to the scientific research development, which has produced knowledge to respond to the profession and society requirements. Knowledge dissemination is essential to produce the transfer of this knowledge to nursing practice; however there are limitations and problems in these stages of the process that must be overcome to make this knowledge allowed to be contrasted with the care provided to individuals, families and communities of human society.
\end{abstract}

Key words: Nursing, knowledge generation, knowledge dissemination, knowledge management, knowledge translation.

Fecha recepción: 22/05/14 Fecha aceptación: 27/04/15

\footnotetext{
*Enfermera. Estudiante Programa Doctorado en Enfermería Universidad de Concepción, Chile. E-mail: ma.elena.lagos@ gmail.com. Fono: (56) (41)2207065.

${ }^{* *}$ Enfermera. Departamento de Enfermería Universidad de Concepción, Chile. E-mail: tparavic@udec.cl. Correspondencia: Dra. Tatiana Paravic Klijn.
} 


\section{INTRODUCCIÓN}

En la era de la globalización del siglo XXI, caracterizado por la evolución de la sociedad humana, la interdependencia y la internacionalización de los procesos de producción y consumo han convertido el conocimiento en un factor de producción y en un elemento esencial de la competitividad, donde la riqueza social es fruto del conocimiento, la ciencia y la tecnología $(1,2)$.

El conocimiento se transforma en la materia prima que se utiliza como un medio para la generación, procesamiento, y distribución de los pensamientos de los países y organizaciones (2). En relación a la generación del conocimiento, la difusión del mismo se ha convertido en una necesidad humana esencial, un derecho, un fundamento de toda organización social. Contribuir a una sociedad informada es uno de los desafíos globales, es un compromiso universal, que busca encausar el potencial de información, el conocimiento y la tecnología para mejorar la calidad de vida de la humanidad; destacando que una de las actividades integradas a la investigación científica es la comunicación de nuevos conocimientos, cuya difusión resulta imprescindible para que se alcance la finalidad: el progreso de la ciencia (1).

Sin embargo, la Organización Mundial de la Salud señala que "existe una brecha entre los avances científicos actuales y su aplicación en la práctica diaria" (3). Con respecto a esto se puede afirmar que la transferencia del conocimiento no se ha producido de manera segura y eficaz. Esto resulta complejo para la disciplina, ya que la transferencia del conocimiento es imprescindible para la práctica de enfermería, sin este medio no se puede hacer la transmisión, recepción y contraste de los cuidados que son y serán proporcionados a las personas, familias y comunidades de esta sociedad humana.

Frente a la transferencia del conocimiento de enfermería, De Pedro refiere: "Lamenta- blemente, no podemos afirmar con determinación que la transferencia de la evidencia sea una prioridad en el ámbito de los cuidados, más bien podríamos decir que existen serias dudas sobre si entienden su necesidad, tanto los propios profesionales de la enfermería como las organizaciones donde éstos desarrollan su práctica. No obstante la comunidad científica ha demostrado sobrado interés por entender la complejidad que rodea la transferencia del conocimiento a la práctica clínica, siendo muchos los estudios que han intentado dar a conocer las diferentes variables que intervienen en la transferencia e implementación de los resultados de la investigación por parte de las enfermeras en su práctica clínica, e identificar cuáles son las dificultades para transferir los nuevos conocimientos" (4).

Por ende este trabajo tiene como objetivo analizar y reflexionar sobre los aspectos relacionados con la generación, difusión y transferencia del conocimiento de enfermería a la práctica del cuidado de Enfermería en Chile.

\section{DESARROLLO}

La generación del conocimiento parte con la expresión mínima de un dato o contenido de un tema, los cuales solo describen una parte de la realidad, no proporcionan juicios ni valores, por lo tanto no son decisivos para la acción, pero sí son importantes para la generación de información (1).

Por su parte la información proviene de la palabra informar o dar forma, el cual se convierte en un recurso social, organizacional que se transmite y cuyo propósito tiene relevancia y significado; es considerada como el recurso base para la adquisición del conocimiento (2).

El conocimiento en sí mismo es la capacidad de convertir la información en acciones efectivas, es una elaboración personal, fruto de las asociaciones del cerebro, a partir de las 
experiencias y la información que poseemos y que se ha seleccionado y analizado, cuyo propósito es dar respuesta a las interrogantes de interés de las personas (1).

De este modo, durante los siglos XX y XXI, la generación del conocimiento para la disciplina de enfermería ha tomado un papel fundamental, permitiendo clarificar y determinar dentro de sus límites: qué tipo de conocimientos buscamos, qué valores tenemos, qué hacemos, creemos y compartimos los miembros de la comunidad de enfermería para que podamos también ser entendidos por otras comunidades disciplinares $(5,6)$.

Por tanto, podemos afirmar que enfermería ha evolucionado como ciencia y disciplina, gracias al desarrollo de la investigación científica, la cual ha generado el conocimiento para dar respuesta a las necesidades de la profesión y de la sociedad. Esta incursión por la investigación ha significado un gran salto para la práctica de enfermería, ya que ha aportado en la comprobación de la práctica diaria, a través de la enfermería basada en la evidencia (5-9).

Según Valenzuela: "En la actualidad enfermería se encuentra en proceso de fortalecimiento de su conocimiento científico para su eficacia y asegurar la calidad metodológica del conocimiento" (10).

Una de las entidades que por excelencia es preferentemente productora y divulgadora de la ciencia, tecnología e innovación, es la Universidad, donde una de sus actividades corresponde a investigación científica (11). En adición a esto, Paravic y Burgos refieren: "La academia representa un papel protagónico en la formación de personas en el área investigativa y los programas de postgrado son responsables de la mayor parte de la producción científica, con la finalidad de formar profesionales altamente calificados" (12). Los estudios de postgrado, dada su propia naturaleza, son programas dentro de los cuales la investigación científica tiene un lugar central $y$, por lo tanto, estimulan el desarrollo de la ciencia, con potencial para incidir en la pro- ducción de tecnología e innovación, donde los aportes logrados deberán quedar plasmados en los artículos y las tesis validadas para el otorgamiento de los grados académicos de magíster y de doctorado.

En virtud de la generación del conocimiento, la difusión del conocimiento se transforma en una necesidad humana esencial, un derecho, un fundamento de toda organización social. Contribuir a una sociedad informada es uno de los desafíos globales, es un compromiso universal, que busca dirigir el contenido de la información, el conocimiento y la tecnología para mejorar la calidad de vida de la humanidad $(2,13,14)$.

La difusión del conocimiento se hace cada vez más importante para la sociedad y para quienes se dedican a la investigación, enseñanza y comunicación en diversas áreas científicas. Los medios por los cuales las personas intercambian ideas se han dado paso desde rudimentarias formas de alfabeto hasta la llegada de la imprenta, el teléfono, el cine, la radio y la televisión. Estos cambios tecnológicos se han producido por la evolución de la sociedad humana $(1,2)$.

Los métodos por los cuales se entrega o difunde la producción del conocimiento son:

- Canales informales o literatura gris: corresponde a los informes, recomendaciones, ponencias no publicadas, traducciones no publicadas, documentos oficiales.

- Canales de información formales o literatura científica: corresponde a libros, capítulos, publicaciones seriadas, periódicos, revistas, memorias, anuarios (2).

Cabe destacar que la "cultura impresa sigue existiendo, pero se ha desarrollado una cultura digital que aporta otros instrumentos y requiere diferentes habilidades y destrezas. Internet facilita el intercambio de información científica por su facilidad técnica, inmediatez, agilidad, economía y por la posibilidad de utilizar sistemas multimedia". "Las publicaciones científicas desempeñan un 
papel fundamental en el desarrollo científico-técnico. Son el punto de partida de cualquier investigación, pues aportan el estado del conocimiento en un tema determinado y son el canal por el que se dará a conocer la investigación concluida" (2).

En el escenario de enfermería del siglo XXI, la difusión del saber de la disciplina ha tenido una serie de problemas que son propios de este colectivo, tales como: dificultades en la redacción de artículos; escasez de revistas científicas en algunos países; revistas que son consideradas onerosas por el colectivo; inexistencia de hemerotecas de enfermería en instituciones de salud para consulta, lo que dificulta además enormemente la enfermería basada en la evidencia; escasez de tiempo para leer las revistas y artículos de reciente publicación y no otorgamiento de parte del propio colectivo de la importancia que tiene la investigación disciplinar, lo que va dando pie para que otros tampoco la consideren importante, lo que termina invisibilizándose en las diversas instituciones de salud. Por lo que podemos decir que los canales de difusión del conocimiento utilizados por la disciplina no han sido de impacto para la colectividad de enfermeras/os. Esto induce a que la práctica de enfermería sea otorgada de forma rutinaria y mecánica, impuesta, desprovista de fundamentación e independencia.

Otro eslabón de la cadena del conocimiento es su transferencia, el cual es imprescindible para que el saber generado exista y sea utilizado por todos quienes realizan aportes al desarrollo del país en lo social, sanitario, político y económico. Desde la perspectiva de la atención sanitaria, específicamente desde el enfoque de los cuidados proporcionados, la transferencia y utilización del conocimiento es trascendental para asegurar una mejor calidad de la atención a las personas, familias y comunidades que se encuentran inmersas en una sociedad que demanda de profesionales, capaces de replantear la forma en la cual se otorgan los cuidados de salud $(15,16)$.

En razón de lo anterior, Davara refiere que "cuando una organización acumula cierta cantidad de conocimiento (o saber) parece lógico que trate de compartirlo e impartirlo, pues de esta forma obtendrá rendimientos de todo tipo (económicos, sociales, profesionales, etc.), constituyéndose en uno de los referentes en su dominio de excelencia, sin olvidar que la interacción con otras organizaciones al transferir el conocimiento tendrá entre otras consecuencias la de aumentar el suyo propio" (15).

Sin embargo, a pesar de lo indispensable que resulta para la sociedad y organizaciones el suministro de conocimiento, lograr que éste sea utilizado por quienes desarrollan la práctica es un gran desafío, que requiere de la participación de los individuos que desarrollan la evidencia y de las personas que ejecutan la práctica para poder entregar de forma eficaz el saber que se ha desplegado a la práctica cotidiana.

En relación a esto, De Pedro, citado por Santillán, refiere que "generar evidencia sin transferirla tan solo nos puede meter en un círculo de frustración a medio y largo plazo, estamos emplazados a resolver este bucle y para ello comenzamos a tener instrumentos que nos permiten el diagnóstico y disponemos de modelos que guíen nuestra actuaciones para mejorar nuestra eficacia", no perdamos nunca de vista que "sin una transferencia garantizada del mejor conocimiento disponible no hay evidencia" (16).

En salud, las enfermeras/os constituyen el grupo más numeroso de profesionales de la salud y por lo tanto el cuidado proporcionado por éstos influye en los resultados del paciente/usuario (17-19). Sin embargo, enfermeras/os, como otros profesionales, a menudo no incorporan los resultados de la investigación en sus prácticas. En relación a lo anterior, existe evidencia de que los resultados proporcionados a través de la investigación no son usados, reportándose entre un 30 a $40 \%$ de usuarios que no reciben atención de acuerdo a la actual evidencia científica; lo que aún es peor, un 20 - $25 \%$ de pacientes 
está en riesgo de recibir atención potencialmente dañina (20).

Durante el transcurso del desarrollo de la disciplina de enfermería se ha dado trascendental importancia al conocimiento generado desde la investigación de enfermería, sin embargo muchas de las investigaciones realizadas en las cuales se invierten múltiples recursos de tipo humano y financiero, no son conocidas ni utilizadas por el colectivo de profesionales de enfermería que desarrollan el cuidado diario de las personas (17-21). Al mismo tiempo, estudios revelan que se han desarrollado investigaciones con la finalidad de sustentar las prácticas médicas, mientras que las investigaciones de enfermería para sustentar la práctica clínica no han sido bien representadas o incorporadas a los planes de cuidados de la profesión, lo cual se ha evidenciado en diferentes estudios, acerca del uso de las investigaciones en la práctica de enfermería (20). Algunos de estos antecedentes es que la investigación de enfermería se ha centrado principalmente en la ejecución de la investigación. Por otra parte podemos mencionar que son muy pocos los documentos que examinan el uso del conocimiento generado en relación a la toma de decisión en la práctica clínica, lo cual se ha demostrado, a través de un estudio realizado al personal de enfermería en Inglaterra el año 2010, arrojando que "no es el conocimiento generado por los investigadores el que dificulta la utilización de éste en la práctica clínica, sino que es el medio por el cual se proporciona la información, en concreto los textos y artículos no son vistos como útiles para el enfermero/a que toma decisiones clínicas" (21).

En la actualidad los desafíos de los equipos de investigación en enfermería es aunar la participación de las instituciones de la salud donde se desarrollan las prácticas clínicas, esto con la finalidad de mejorar la transferencia del conocimiento desde la academia a la práctica del cuidado (22-24). Por otra parte el conocimiento generado en las investigaciones debe ser capaz de satisfacer las necesidades de la práctica de enfermería cotidiana y no sólo quedarse en abstracciones que solo los investigadores o académicos pueden interpretar.

De este modo la transferencia del conocimiento es de responsabilidad de los investigadores, académicos, profesionales e instituciones de salud, con el propósito de que el conocimiento generado sea conocido por las enfermeras/os y demás profesionales de la salud y utilizado por los equipos de salud, para de esta forma conseguir una atención de calidad para las personas, familias y comunidades.

La necesidad de que el conocimiento generado sea utilizado por quienes desarrollan la práctica de enfermería, hace que sea inevitable mediar intervenciones para que no solo baste con proponer que el conocimiento sea conocido o transferido a la práctica clínica, sino que éste debe ser adecuado a la comprensión de quienes desarrollan la labor de cuidados de enfermería. En relación a ello, Trentini refiere que "para concretizar tal transferencia de conocimiento teórico para la práctica asistencial, hay que considerar que la migración del conocimiento no corresponde simplemente en el traslado del mismo, tal cual fue concebido por los investigadores, él precisa ser reconstruido, o sea, necesita de un proceso de des-abstracción, de modo que pueda ser refinado y ajustable a las condiciones de la práctica. Para efectuar el refinamiento del conocimiento, los investigadores necesitan escuchar la voz de la práctica, ya que es en el campo de la práctica que las formulaciones teóricas emergen y precisan de la práctica para retornar, con el fin de ser testadas, absorbidas e incorporadas o refutadas" (24).

Indiscutiblemente enfermería necesita y depende como profesión de la generación de nuevos conocimientos, de la difusión y de la articulación del conocimiento generado con la práctica clínica, para que de este modo se transfiera y se produzca la evolución de la disciplina de enfermería. 
Existen propuestas para mejorar la transferencia del conocimiento, entre ellas "la formación de grupos de investigación, quienes incorporan actividades administrativas que favorecen esta transferencia, tales como: proveer recursos humanos y materiales suficientes en número y calidad, incentivar actitudes de compromiso con la transferencia, tanto individual como organizacional y elaborar un plan de educación, a largo plazo, para la capacitación profesional. Además de esos aspectos, exista disponibilidad de metodologías de tipo participativa, por ejemplo la investigación-acción y la investigación convergente-asistencial, que se muestran apropiadas para la simultaneidad de la construcción de conocimiento y su transferencia para la práctica" (24). Las autoras del presente artículo agregan a esta propuesta la integración docente-asistencia; ir a los campos clínicos y conversar con las/ los enfermeras/os y usuarios/as de los servicios realizando un levantamiento de los problemas detectados, fomentar la creación de grupos de enfermeros/as investigadores que acopien las diversas evidencias para tenerlas a disposición de las enfermeras/os clínicos de los diversos servicios; enseñar desde el primer año en la universidad que una atención de calidad es aquella basada en la mejor evidencia, desarrollando en el/ la estudiante la conducta de buscar las evidencias, para que cuando trabajen en los servicios de salud sea ésta una práctica incorporada a sus modus operandi y otorgue a los conocimientos producidos el lugar que se merecen "en la práctica".

Laufer refiere que "es responsabilidad de toda la sociedad, pero más aún de los docentes como formadores de conocedores, y de los investigadores, como creadores del conocimiento, asegurar que la información creada sea suficiente, correcta y que consiga alcanzar a la mayor cantidad posible de personas" (25).

\section{CONSIDERACIONES FINALES}

Enfermería ha evolucionado como ciencia y disciplina gracias al desarrollo de la investigación científica, la cual ha generado el conocimiento para dar respuesta a las necesidades de la profesión y de la sociedad. Las actividades integradas a la investigación científica de enfermería han sido la fuente principal para la generación de nuevos conocimientos, donde se han invertido múltiples recursos financieros y humanos. La difusión resulta imprescindible para que el saber generado alcance la finalidad y el progreso de la ciencia de enfermería. Esta incursión en la investigación ha significado un gran salto para la práctica de enfermería, ya que ha aportado en la comprobación de la práctica diaria, a través de la enfermería basada en la evidencia. Sin embargo, sin una adecuada difusión del conocimiento no se logrará la transferencia al colectivo de profesionales de enfermería y de los equipos multidisciplinarios que presta atención sanitaria, quedando de manifiesto que no se obtendrá el más alto estándar de calidad en la salud y en el cuidado de las personas.

La necesidad de que el conocimiento generado sea utilizado por quienes desarrollan la práctica de enfermería, hace que sea preciso mediar intervenciones para que no sólo convenga con proponer que el conocimiento sea conocido, sino que transferido a la práctica clínica, acomodándolo a la comprensión de quienes desarrollan la labor de cuidados de enfermería en la práctica clínica concreta. Con la finalidad de mejorar el cuidado de enfermería, en la actualidad se han propuesto desafíos para integrar la participación de las instituciones de la salud donde se desarrollan las experiencias clínicas, esto con la finalidad de mejorar la transferencia del conocimiento desde la academia a la práctica del cuidado. Por otra parte, el conocimiento generado 
de las investigaciones debe ser capaz de satisfacer las necesidades de la experiencia de enfermería cotidiana y no sólo quedarse en abstracciones que solo los/las investigadores/ as o académicos/as pueden interpretar.

\section{REFERENCIAS}

1. Middleton M. De la gestión de la información a la gestión del conocimiento: perspectivas sobre el desarrollo. El profesional de la información [Internet]. 1999 [citado 10 agosto 2013]. Disponible en: http://www.elprofesionaldelainformacion.com/contenidos/1999/mayo/html

2. Vilches C. La información y difusión del conocimiento [Internet]. Primer taller para editores y autores científicos; 6-8 diciembre 2006; La Paz, Bolivia: Sistema Regional de Información en Línea para Revistas Científicas de América Latina, el Caribe, España y Portugal - Latindex; [citado 20 agosto 2013]. 2006. Disponible en: http://es.slideshare.net/Latindex/ informacin-y-difusion-del-conocimiento-vilches

3. World Health Organizaion. Department of Knowledge management and sharing. The Know-do Gap. Knowledge Translation in Global Health; 10-12 Oct 2005; Geneva: WHO; 2005.

4. De Pedro Gómez J. Sin transferencia no hay evidencia. Evidentia [Internet]. 2011 [citado 20 agosto 2013]; 8(35). Disponible en: http://www.index-f.com/evidentia/n35/ev3506.php.

5. Urra E. Avances de la ciencia de enfermería y su relación con la disciplina. Cienc. enferm. 2009; XV(2): 9-18.

6. Aimar A, Videla N, Torres M. Tendencias y perspectivas de la ciencia de enfermería. Enferm. glob. [Internet]. 2006 [citado 10 agosto 2013]; 5(2). Disponible en: http:// revistas.um.es/eglobal/article/view/385
7. Ramírez-Elizondo N, Paravic Klijn T. Enfermería basada en la evidencia, una ruta hacia la aplicación en la práctica profesional. Enfermería en Costa Rica [Internet]. 2011 [citado 10 agosto 2013]; 32(2). Disponible en: http://revista.enfermeria. cr/ediciones/revista-enfermeria-en-costa-rica-volumen-3222011

8. Eterovic C, Stiepovich J. Enfermería basada en la evidencia y formación profesional. Cienc. enferm. 2010; XVI(3): 9-14.

9. González M. Interiorizar la EBE, asumir la práctica basada en evidencias. Evidentia [Internet] 2005 [citado 20 agosto 2013]; 2(4). Disponible en: http://www. index-f.com/evidentia/n4/101articulo. php

10. Valenzuela S. Desde la "transferencia" a la "traducción" del conocimiento en enfermería. Cienc. enferm. 2007; XIII(1): 7-7.

11. Herrera R, Gutiérrez J, eds. Conocimiento, innovación y desarrollo [Internet]. San José, Costa Rica: Impresión Gráfica del Este; 2011 [citado 10 agosto 2013]. 290 p. Disponible en: http://catedrainnovacion.ucr.ac.cr/librocid.pdf

12. Paravic T, Burgos M. Evaluación de la calidad de los resúmenes de tesis de un programa de Magíster en enfermería. Cienc. enferm. 2009; XV(3): 55-68.

13. Organización de Estados Iberoamericanos para la Educación, la Ciencia y la Cultura. Corporación Escenarios (Org). United Nations, Economic Commission for Latin America and the Caribbean. Educación y globalización: Los desafíos para América Latina; Globalización, ciencia y tecnología. Vol II. Bogotá: Organización de Estados Iberoamericanos para la Educación, la Ciencia y la Cultura (OEI): Naciones Unidas, CEPAL: Corporación Escenarios; 2004, 206 p.

14. Zozaya V. Las redes y la transferencia de Conocimiento. Boletín GC: Gestión Cultural [Internet]. 2006 [citado 20 agosto 2013]; 14: 3-9. Disponible en: http:// 
www.gestioncultural.org/boletin/2006/ bgc14-vzozaya.pdf.

15. Davara F. Transferencia del conocimiento. 24 marzo 2007 [citado 20 agosto 2013]. En: Tendencias 21. Ciencia, tecnología, sociedad y cultura [blog en Internet]. Madrid: Zine Consultores S.L. Disponible en: http://www.tendencias21. net/conocimiento/Transferencia-del-Conocimiento-I_a5.html

16. Santillán A. La falta de transferencia nos deja sin evidencias. 20 enero 2012 [citado 10 agosto 2013]. En: Enfermería basada en la evidencia [blog en Internet]. España: [editorial desconocida]; 2012; Disponible en: ebevidencia.com/archivos/26

17. Orellana A, Paravic T. Enfermería basada en la evidencia. Barreras y estrategias para su implementación. Cienc. enferm. 2007; XIII(1): 17-24.

18. Estabrooks CA, Scott $S$, Squires JE, Stevens B, O'Brien-Pallas L, Watt-Watson J, et al. Patterns of research utilization on patient care units. Implement Sci [Internet]. 2008 [citado 19 agosto 2013]; (2) 3:31. Disponible en: http://www.ncbi. nlm.nih.gov/pubmed

19. Hagedorn H, Hogan M, Smith J, Bowman C, Curran G, Espadas D, et al. Lessons Learned about Implementing Research Evidence into Clinical Practice Experiences from VA QUERI. J Gen Intern Med. 2006; (21): S21-24.

20. Thompson DS, Estabrooks C, Scott-Findlay S, Moore K, Wallin L. Interventions aimed at increasing research use in nursing: A systematic review. Implement Sci [Internet]. 2007 [citado 10 agosto 2013]; 2:15. Disponible en: http://www.implementationscience.com/content/2/1/15

21. Thompson C, McCaughan D, Cullum N, Sheldon TA, Mulhall A, Thompson DR. Research information in nurses' clinical decision-making: What is useful? J Adv Nurs. 2001; 36(3): 376-88.

22. González-Torrente S, Pericas-Beltrán J, Bennasar-Veny M, Adrover-Barceló $\mathrm{R}$, Morales-Asencio JM, De Pedro-Gómez J. Perception of evidence-based practice and the professional environment of primary health care nurses in the Spanish context: a cross-sectional study. BMC Health Serv Res [Internet]. 2012 [citado 2 agosto 2013]; 12: 227. Disponible en: http://www.biomedcentral.com

23. Phillips J. Knowledge is power: using nursing information management and Leadership interventions to improve services to patients, clients and users J Nurs Manag. 2005; 13(6): 524-536.

24. Trentini M. Grupos de investigación en enfermería: de la transferencia del conocimiento a la práctica. Texto contexto-enferm. 2012; 21(4): 727-8.

25. Laufer M. La difusión del conocimiento. Interciencia [Internet]. 2008 [citado 2 agosto 2013]; 33(11): 786. Disponible en: http://www.redalyc.org/articulo. oa?id=33913601. 